\title{
Simultaneous Diophantine approximation on the circle and Hausdorff dimension
}

\author{
By H. DICKINSON AND M. M. DODSON \\ Department of Mathematics, University of York, York YO10 5DD. \\ (Received 21 September 1999; revised 2 February 2000)
}

The functional relations between the coordinates of points on a manifold make the study of Diophantine approximation on manifolds much harder than the classical theory in which the variables are independent. Nevertheless there has been considerable progress in the metric theory of Diophantine approximation on smooth manifolds. To describe this, some notation and terminology are needed. Given $\mathbf{x} \in \mathbb{R}^{n}$, let $|\mathbf{x}|=\max \left\{\left|x_{1}\right|, \ldots,\left|x_{n}\right|\right\}$ be the height of $\mathbf{x}$, let $M$ be an $m$-dimensional manifold embedded in $\mathbb{R}^{n}$ and let $\psi: \mathbb{N} \rightarrow \mathbb{R}^{+}$converge to 0 at infinity. For each $t \in \mathbb{R}$, let

$$
\|t\|=\min \{|t-r|: r \in \mathbb{Z}\}=\operatorname{dist}(t, \mathbb{Z}),
$$

the distance of $t$ from $\mathbb{Z}$, and for each $\mathbf{x}=\left(x_{1}, \ldots, x_{n}\right) \in \mathbb{R}^{n}$, let

$$
\|\mathbf{x}\|=\max \left\{\left\|x_{1}\right\|, \ldots,\left\|x_{n}\right\|\right\} .
$$

Points in the set

$$
\mathscr{S}(M ; \psi)=\{\mathbf{x} \in M:\|q \mathbf{x}\|<\psi(q) \text { for infinitely many } q \in \mathbb{N}\}
$$

are called simultaneously $\psi$-approximable. When $\psi(q)=q^{-v}$, we denote the set $\mathscr{S}(M ; \psi)$ by $\mathscr{S}_{v}(M)$, so that

$$
\mathscr{S}_{v}(M)=\left\{\mathbf{x} \in M:\|q \mathbf{x}\|<q^{-v} \quad \text { for infinitely many } q \in \mathbb{N}\right\} .
$$

The manifold $M \subset \mathbb{R}^{n}$ is termed extremal if the Lebesgue measure of $\mathscr{S}_{v}(M)$ induced on $M$ is 0 for each $v>1 / n$. There is an equivalent 'dual' definition of extremality, arising from Khintchine's Transference Principle. Let

$$
\mathscr{L}(M ; \psi)=\left\{\mathbf{x} \in M:\|\mathbf{q} \cdot \mathbf{x}\|<\psi(|\mathbf{q}|) \quad \text { for infinitely many } \mathbf{q} \in \mathbb{Z}^{n}\right\}
$$

and

$$
\mathscr{L}_{v}(M)=\left\{\mathbf{x} \in M:\|\mathbf{q} \cdot \mathbf{x}\|<|\mathbf{q}|^{-v} \quad \text { for infinitely many } \mathbf{q} \in \mathbb{Z}^{n}\right\} .
$$

Then $M$ is extremal if the induced measure of $\mathscr{L}_{v}(M)$ is 0 for $v>n$. Kleinbock and Margulis have shown in [12] that manifolds $M$ embedded in $\mathbb{R}^{n}$ which are 'nondegenerate' almost everywhere on $M$ (i.e. everywhere except for a set of induced Lebesgue measure zero on $M$ ) and which are smooth (i.e. with $C^{k}$ local parametrisation functions for certain $k \in \mathbb{N}$ ) are extremal (in fact they showed that such manifolds are strongly extremal). Non-degeneracy can be regarded as a local "nonflatness' condition; further details are in [12]. Note that by Dirichlet's theorem, $\mathscr{S}_{v}(M)=M$ when $v \leqslant 1 / n$ and $\mathscr{L}_{v}(M)=M$ when $v \leqslant n$.

Hausdorff dimension provides a way of distinguishing between sets of measure 0 
(see $[10,13]$ for further information). It is known that when $M$ is a $C^{3}$ planar curve with non-zero curvature everywhere except on a set of Hausdorff dimension zero, the Hausdorff dimension $\operatorname{dim} \mathscr{L}_{v}(M)$ of $\mathscr{L}_{v}(M)$ is given by

$$
\operatorname{dim} \mathscr{L}_{v}(M)=\frac{3}{v+1}
$$

for $v \geqslant 2$ [3]. It is also known that for $m$-dimensional $C^{3}$ manifolds $M$ where $m \geqslant 2$ and for which two principal curvatures do not vanish (this condition reduces to Gaussian curvature for surfaces in 3 dimensions) except on a set of Hausdorff dimension $m-1$, the Hausdorff dimension of $\mathscr{L}(M ; \psi)$ is $m-1+(n+1) /(\lambda+1)$ when $\psi$ is monotonically decreasing and $\lambda \geqslant n$, where $\lambda=\liminf _{N \rightarrow \infty}(\log (1 / \psi(N)) /(\log N))$, the lower order of $1 / \psi$ at infinity [6]. This dimension is a lower bound when $M$ is extremal and $C^{1}[5]$.

Not much, however, is known about the Hausdorff dimension of the set $\mathscr{S}(M ; \psi)$, although a Khintchine-type theorem holds for 2-convex $C^{3}$ manifolds [8] and an asymptotic formula (which is a stronger version of the Khintchine-type theorem) holds under fairly restrictive curvature conditions [9]. Only estimates for the Hausdorff dimension of the set $\mathscr{S}_{v}(M)$ are known (see [4] for further details and references). Melnichuk [14] studied Diophantine approximation on the unit circle $\mathbb{S}^{1}=$ $\left\{(x, y) \in \mathbb{R}^{2}: x^{2}+y^{2}=1\right\}$ and obtained estimates for the Hausdorff dimension of the set

$$
\begin{aligned}
\mathscr{S}_{v}\left(\mathbb{S}^{1}\right) & =\left\{\mathbf{x} \in \mathbb{S}^{1}:\|q \mathbf{x}\|<q^{-v} \quad \text { for infinitely many } q \in \mathbb{N}\right\} \\
& =\left\{(x, y) \in \mathbb{S}^{1}: \max \{\|q x\|,\|q y\|\}<q^{-v} \quad \text { for infinitely many } q \in \mathbb{N}\right\} .
\end{aligned}
$$

He used exponential sums to obtain estimates for the case $\frac{1}{2}<v \leqslant 1$. By applying Khintchine's Transference Principle to Baker's general result (2), one can show that

$$
\frac{3(1-v)}{1+v} \leqslant \operatorname{dim} \mathscr{S}_{v}(M) \leqslant \frac{3}{2(1+v)},
$$

when $M$ is a planar curve satisfying the conditions specified above. Thus these estimates hold for $M=\mathbb{S}^{1}$.

In the case when $v>1$, Melnichuk used Pythagorean triples to show that

$$
\frac{1}{2(1+v)} \leqslant \operatorname{dim} \mathscr{S}_{v}\left(\mathbb{S}^{1}\right) \leqslant \frac{1}{1+v} .
$$

(Non-zero integers $p, r, q$ satisfying $p^{2}+r^{2}=q^{2}$ are called a Pythagorean triple [1].) The usual covering argument gives the upper bound and he used regular systems [2] and Dirichlet's theorem for the lower bound. In this paper we use ubiquity together with Lemma 2 below to establish the lower bound $1 /(v+1)$ in (4), thus obtaining the exact Hausdorff dimension for $v>1$. Ubiquity and regular systems are essentially equivalent on $\mathbb{R}$ but the former gives the Hausdorff dimension more directly. However, the latter can also be used with Lemma 2 to obtain the same lower bound.

Theorem 1. For $v>1$

$$
\operatorname{dim} \mathscr{S}_{v}\left(\mathbb{S}^{1}\right)=\frac{1}{v+1} .
$$

As far as we know, this is the first reasonably complete non-trivial result for the Hausdorff dimension of the set $\mathscr{S}_{v}(M)$ for a smooth manifold $M$ in $\mathbb{R}^{n}$ when $v$ is 
larger than the extremal value of $1 / n$. Melnichuk's paper [14] is in Russian and is not readily available so his proof for the upper bound will be included for completeness.

\section{Proof of Theorem 1}

The key to the theorem is that rational points $(p / q, r / q)$ which approximate a point $(x, y)$ on the unit circle sufficiently closely must be on the circle.

Lemma 1. Suppose the point $(x, y) \in \mathbb{S}^{1}$ satisfies the inequalities

$$
|q x-p|,|q y-r|=o(1 / q)
$$

for $p, q, r \in \mathbb{Z}, q \geqslant 1$. Then for $q$ sufficiently large, the point $(p / q, r / q) \in \mathbb{S}^{1}$.

Proof. Let $(x, y) \in \mathbb{S}^{1}$ so that $x^{2}+y^{2}=1$. Assume that there exist $p, r \in \mathbb{Z}, q \in \mathbb{N}$ such that $q x-p=\varepsilon$ and $q y-r=\varepsilon^{\prime}$ for some $\varepsilon, \varepsilon^{\prime} \in \mathbb{R}$ with $|\varepsilon|,\left|\varepsilon^{\prime}\right|=o(1 / q)$. Then $q^{2}\left(1-x^{2}\right)=\left(r+\varepsilon^{\prime}\right)^{2}, q^{2} x^{2}=(p+\varepsilon)^{2}$ and $|p|=O(q),|r|=O(q)$. On rearranging it is readily verifed that

$$
q^{2}=p^{2}+r^{2}+2 p \varepsilon+2 r \varepsilon^{\prime}+\varepsilon^{2}+\varepsilon^{\prime 2} .
$$

But

$$
\left|q^{2}-p^{2}-r^{2}\right|=\left|2 p \varepsilon+2 r \varepsilon^{\prime}+\varepsilon^{2}+\varepsilon^{\prime 2}\right|=O(q) o(1 / q)=o(1)<1
$$

for $q$ large enough. As $p, q, r \in \mathbb{Z}$, this implies that $q^{2}=r^{2}+p^{2}$, whence the point $(p / q, r / q)$ lies on the circle.

When $p, r$ are coprime and $p$ is even the Pythagorean triples $(p, r, q)$ are generated by positive coprime integers $a, b$ of the following form:

$$
p=2 a b, \quad r=a^{2}-b^{2}, \quad q=a^{2}+b^{2}
$$

(see [1] or [11]). Let $V(\theta ; Q)$ be a sector in the disc of radius $Q^{\frac{1}{2}}$ subtending an angle $\theta$ at the origin. The number of lattice points $(a, b)$ of opposite parity (i.e. $a, b$ are not both even or both odd) in $V(\theta ; Q)$ is comparable to $\theta Q$ (the number is $\theta Q(1+o(1)) / 4$ ). By using the Möbius function, it can be seen that the number $\mathscr{N}(V(\theta ; Q))$ of such lattice points $(a, b)$ for which $a$ and $b$ are also coprime is comparable to $\theta Q$. Indeed

$$
\mathscr{N}(V(\theta ; Q))=3 \theta Q(1+o(1)) / 2 \pi^{2} \asymp \theta Q,
$$

where for positive $a, b, a \asymp b$ indicates $a=O(b)$ and $b=O(a)$ i.e., that $a$ and $b$ are comparable. The points $(p / q, r / q),(r / q, p / q)$, where $p$ and $r$ are coprime to $q$, in $\mathbb{S}^{1} \cap[0,1]^{2}$ and satisfying (5) are in one-to-one correspondence with lattice points $(a, b)$ where $a, b$ are coprime positive integers of opposite parity with $a>b$.

\subsection{The upper bound}

In determining the Hausdorff dimension of $\mathscr{S}_{v}\left(\mathbb{S}^{1}\right)$, it is more convenient to work with an auxiliary subset of the line. Let $A=\left\{(x, y) \in \mathbb{S}^{1}:|x| \leqslant y\right\}$ be the arc of $\mathbb{S}^{1}$ subtending an angle of $\pi / 2$ and symmetric about the vertical axis. Then $\mathscr{S}_{v}\left(\mathbb{S}^{1}\right)$ is the union of $\mathscr{S}_{v}\left(\mathbb{S}^{1}\right) \cap A$ and its intersection with the three rotations of $A$ by $\pi / 2$. Since the Hausdorff dimension is not affected by rotation, the Hausdorff dimension of each of the four sets is the same and so

$$
\operatorname{dim} \mathscr{S}_{v}\left(\mathbb{S}^{1}\right)=\operatorname{dim}\left(\mathscr{S}_{v}\left(\mathbb{S}^{1}\right) \cap A\right)=\operatorname{dim} \mathscr{S}_{v}\left(\mathbb{S}^{1} \cap A\right) .
$$


Let $A_{v}$ be the projection of $\mathscr{S}_{v}\left(\mathbb{S}^{1}\right) \cap A$ to the real line, so that

$$
S_{v}=\left\{u \in\left[\frac{-1}{\sqrt{2}}, \frac{1}{\sqrt{2}}\right]:\left\|q\left(u,\left(1-u^{2}\right)^{\frac{1}{2}}\right)\right\|<q^{-v} \quad \text { for infinitely many } q \in \mathbb{N}\right\} .
$$

Any cover of $\mathscr{S}_{v}\left(\mathbb{S}^{1}\right) \cap A$ consisting of squares $\Delta_{i}$ of side length $\ell(\Delta)$ and with sides parallel to the axes can be converted by projection to a cover of $S_{v}$ by intervals $I_{i}$ with $s$-length $\sum_{i} \ell\left(I_{i}\right)^{s}=\sum_{i} \ell\left(\Delta_{i}\right)^{s}$. Moreover any cover of $S_{v}$ by intervals $I_{i}$ can be lifted to a cover of $\mathscr{S}_{v}\left(\mathbb{S}^{1}\right) \cap A$ by squares $\Delta_{i}$ of sidelength $\ell\left(I_{i}\right)$. It follows that

$$
\operatorname{dim} S_{v}=\operatorname{dim} \mathscr{S}_{v}\left(\mathbb{S}^{1}\right) \cap A=\operatorname{dim} \mathscr{S}_{v}\left(\mathbb{S}^{1}\right) .
$$

It is more convenient to work with $S_{v}$ which can be expressed as

$$
S_{v}=\left\{u \in\left[\frac{-1}{\sqrt{2}}, \frac{1}{\sqrt{2}}\right]: u \in B(q) \quad \text { for infinitely many } q \in \mathbb{N}\right\},
$$

where

$$
\begin{aligned}
B(q) & =\left\{u \in\left[\frac{-1}{\sqrt{2}}, \frac{1}{\sqrt{2}}\right]:\left\|q\left(u, \sqrt{1-u^{2}}\right)\right\|<q^{-v}\right\} \\
& \subset \bigcup_{p=-q}^{q}\left\{u \in\left(\frac{p}{q}-\frac{1}{q^{v+1}}, \frac{p}{q}+\frac{1}{q^{v+1}}\right):\left|\sqrt{1-u^{2}}-\frac{r}{q}\right|<\frac{1}{q^{v+1}} \quad \text { for some } r \in \mathbb{Z}\right\} .
\end{aligned}
$$

The number of positive rationals $p / q$ with $q \leqslant Q$ such that $p, r\left(=\sqrt{q^{2}-p^{2}}\right), q$ is a Pythagorean triple is $\ll Q(\ll$ is the usual Vinogradov notation used instead of $O(Q))$. Taking symmetries into account, the number of distinct rational points $(p / q, r / q)$ in their lowest terms, with $2^{t}<q \leqslant 2^{t+1}$, lying on the circle $\mathbb{S}^{1}$ is

$$
\mathscr{N}\left(2^{t+1}\right)-\mathscr{N}\left(2^{t}\right) \ll 2^{t},
$$

where $\mathscr{N}(N)$ is the number of distinct rational points $(p / q, r / q)$ in their lowest terms lying on $\mathbb{S}^{1}$ with $q \leqslant N$.

Since the length of the interval $\left(p / q-1 / q^{v+1}, p / q+1 / q^{v+1}\right)$ is $2 / q^{v+1} \ll\left(2^{t}\right)^{-v-1}$ and since $p, q$ can be taken to be coprime without loss of generality, $S_{v}$ has a cover of intervals with $s$-length

$$
\ll \sum_{t=0}^{\infty} 2^{-t(v+1) s}\left(\mathscr{N}\left(2^{t+1}\right)-\mathscr{N}\left(2^{t}\right)\right) \ll \sum_{t=0}^{\infty} 2^{-t(v+1) s+t}<\infty
$$

when $s>1 /(v+1)$. Hence by $(7)$,

$$
\operatorname{dim} \mathscr{S}_{v}\left(\mathbb{S}^{1}\right)=\operatorname{dim} S_{v} \leqslant 1 /(v+1)
$$

for $v>1$.

Using the results of Wiles or Faltings, it can be shown that when $k \geqslant 3$ and $\Gamma_{k}$ is the curve $\left\{(x, y): x^{k}+y^{k}=1\right\}$, the set $\mathscr{S}_{v}\left(\Gamma_{k}\right)$ is finite for $v>k-1$ and is $\Gamma_{k}$ when $v \leqslant \frac{1}{2}$. The range $\frac{1}{2}<v<k-1$ is covered by the estimate (3) applied with $M=\Gamma_{k}$. Hence

$$
\operatorname{dim} \mathscr{S}_{v}\left(\Gamma_{k}\right) \begin{cases}=1, & v \leqslant \frac{1}{2}, \\ \leqslant \frac{3}{2(v+1)}, & \frac{1}{2}<v<k-1, \\ =0, & v \geqslant k-1 .\end{cases}
$$


1.2. The lower bound

This will be proved using the modification [15] of ubiquity introduced in [7]. The definition we now give is simpler than the general one in [7] but is equivalent for affine spaces and in particular for intervals with resonant sets consisting of points.

Let $\Omega$ be a non-empty open set in Euclidean space, let $R$ be a measurable subset of $\Omega$ and let $|R|$ be the Lebesgue measure of $R$. For each $\delta>0$, let

$$
B(R ; \delta)=\{\mathbf{x} \in \Omega: \operatorname{dist}(\mathbf{x}, R)<\delta\},
$$

where $\operatorname{dist}(\mathbf{x}, R)=\inf \{|\mathbf{x}-\mathbf{y}|: \mathbf{y} \in R\}$. Let $\mathscr{R}=\left\{R_{j}: j \in J\right\}$ be a family of affine subsets of $\Omega$, where $J$ is an index set with each $j \in J$ having a weight $\lfloor j\rfloor>0$ and each $R_{j}$ having the same dimension $\operatorname{dim} \mathscr{R}$ and codimension $\operatorname{codim} \mathscr{R}=\operatorname{dim} \Omega-\operatorname{dim} \mathscr{R}$. The family $\mathscr{R}$ is said to be ubiquitous with respect to a function $\rho: \mathbb{N} \rightarrow \mathbb{R}^{+}$if there exists a constant $c>0$ such that for any open subset $A \subset \Omega$ and all sufficiently large $N \in \mathbb{N}$,

$$
\left|A \cap\left(\bigcup_{\lfloor j\rfloor=1}^{N} B\left(R_{j}, \rho(N)\right)\right)\right| \geqslant c|A| .
$$

Given a function $\Psi: \mathbb{N} \rightarrow \mathbb{R}^{+}$, denote by $\Lambda(\mathscr{R} ; \Psi)$ the lim-sup set of points in $\Omega$ which lie in infinitely many $B\left(R_{j} ; \Psi(\lfloor j\rfloor)\right)$, i.e.

$$
\Lambda(\mathscr{R} ; \Psi)=\bigcap_{N=1}^{\infty} \bigcup_{k=N}^{\infty} \bigcup_{\lfloor j\rfloor=k} B\left(R_{j} ; \Psi(\lfloor j\rfloor)\right) .
$$

The following lower bound for the Hausdorff dimension of $\Lambda(\mathscr{R} ; \Psi)$ is proved in [7].

Theorem 2. Let $\Psi: \mathbb{N} \rightarrow \mathbb{R}^{+}$be a monotonically decreasing function and suppose that the family $\mathscr{R}$ is ubiquitous with respect to a function $\rho: \mathbb{N} \rightarrow \mathbb{R}^{+}$, where $\rho(N)$ tends to 0 monotonically as $N$ tends to infinity. Then

$$
\operatorname{dim} \Lambda(\mathscr{R} ; \Psi) \geqslant \operatorname{dim} \mathscr{R}+\gamma \operatorname{codim} \mathscr{R},
$$

where

$$
\gamma=\max \left\{1, \limsup _{N \rightarrow \infty} \frac{\log \rho(N)}{\log \Psi(N)}\right\} .
$$

The relationship between the lattice points $(a, b)$ and the corresponding points $(p / q, r / q)$ on the circle $\mathbb{S}^{1}$ has to be discussed further.

Lemma 2. Let $A$ be an open subset of $[\sqrt{15} / 8, \sqrt{3} / 2]$ and let $Q$ be sufficiently large. Then there exists a constant $K>0$ independent of $A, Q$ such that

$$
\left|A \cap \bigcup_{p / q}\left(\frac{p}{q}-\frac{K}{Q}, \frac{p}{q}+\frac{K}{Q}\right)\right| \geqslant K|A|,
$$

where here and elsewhere the union is over rationals $p / q$ with $p, \sqrt{q^{2}-p^{2}}, q$ a Pythagorean triple and $|q| \leqslant Q$.

Proof. Let $W(\theta ; Q)=V(\theta ; Q) \backslash V(\theta ; Q / 2)$, so that $W(\theta ; Q)$ is the set of points $(x, y)$ 
in $\left[0, Q^{\frac{1}{2}}\right]^{2}$ satisfying

$$
Q / 2<x^{2}+y^{2} \leqslant Q, \theta^{\prime} \leqslant \tan ^{-1} y / x \leqslant \theta^{\prime}+\theta
$$

for some $\theta^{\prime} \in[0, \pi / 2-\theta]$. Thus $W(\theta ; Q)$ is a sector of an annulus subtending an angle $\theta$ with area

$$
\theta(Q-Q / 4) \asymp \theta Q .
$$

Hence the number $\mathscr{N}(W(\theta ; Q))$ of lattice points $(a, b) \in \mathbb{Z}^{2} \cap W(\theta ; Q)$, where $a, b$ are of opposite parity and coprime, is comparable to $\theta Q$; in fact, by (6) above,

$$
\mathscr{N}(W(\theta ; Q))=\frac{3 \theta Q}{8 \pi^{2}}(1+o(1))>\frac{\theta Q}{32} .
$$

The set $A$ is a countable union of disjoint open intervals $\left(\alpha_{r}, \beta_{r}\right)$. Choose, as we may, $r_{0} \in \mathbb{N}$ so that $\left|\bigcup_{r=1}^{r_{0}}\left(\alpha_{r}, \beta_{r}\right)\right| \geqslant|A| / 2$. Consider one of these intervals $(\alpha, \beta)$ say. The interval $(\alpha, \beta)$ is the projection of the are in $\mathbb{S}^{1}$ with endpoints $\left(\alpha, \sqrt{1-\alpha^{2}}\right)$ and $\left(\beta, \sqrt{1-\beta^{2}}\right)$. The angle $\theta$ subtended by the arc is comparable to $\beta-\alpha$, i.e.

$$
\theta \asymp \beta-\alpha .
$$

As the lattice points $(a, b)$ are co-prime, each lies on a unique line through the origin of gradient $b / a$. The function $f:[\sqrt{3}, \sqrt{15}] \rightarrow[\sqrt{15} / 8, \sqrt{3} / 2]$ given by

$$
f(x)=\frac{2 x}{1+x^{2}}
$$

takes $b / a$ to $2 a b /\left(a^{2}+b^{2}\right)=p / q$, where $p, \sqrt{q^{2}-p^{2}}, q$ is a Pythagorean triple. The function $f$ is one-to-one since its derivative

$$
f^{\prime}(x)=\frac{2\left(1-x^{2}\right)}{\left(1+x^{2}\right)^{2}}<0
$$

for all $x \in[\sqrt{3}, \sqrt{15}]$. It follows that

$$
\mathscr{N}(W(\theta ; Q)) \asymp \nu(\alpha, \beta),
$$

the number of $p / q \in(\alpha, \beta)$ with $Q / 2<q \leqslant Q$ and $p, \sqrt{q^{2}-p^{2}}, q$ a Pythagorean triple. But by the above, (10) and (11),

$$
\nu(\alpha, \beta) \asymp \mathscr{N}(W(\theta ; Q)) \asymp \theta Q \asymp(\beta-\alpha) Q \geqslant c_{1} Q(\beta-\alpha),
$$

where $c_{1}>0$ is independent of $\alpha, \beta$ and $Q$.

Also the function $f$ is bi-Lipschitz on the interval $[\sqrt{3}, \sqrt{15}]$, so that

$$
\left|f(x)-f\left(x^{\prime}\right)\right| \asymp\left|x-x^{\prime}\right|
$$

for $x, x^{\prime} \in[\sqrt{3}, \sqrt{15}]$. Thus when $a / b \neq a^{\prime} / b^{\prime}$,

$$
\left|f\left(\frac{b}{a}\right)-f\left(\frac{b^{\prime}}{a^{\prime}}\right)\right|=\left|\frac{2 a b}{a^{2}+b^{2}}-\frac{2 a^{\prime} b^{\prime}}{a^{\prime 2}+b^{\prime 2}}\right| \asymp\left|\frac{b}{a}-\frac{b^{\prime}}{a^{\prime}}\right| \geqslant \frac{1}{a a^{\prime}} \geqslant \frac{1}{Q}
$$

since $a, a^{\prime} \leqslant Q^{\frac{1}{2}}$. Moreover if $p / q \neq p^{\prime} / q^{\prime}$, then $a / b \neq a^{\prime} / b^{\prime}$ and

$$
\left|\frac{p}{q}-\frac{p^{\prime}}{q^{\prime}}\right|=\left|f\left(\frac{b}{a}\right)-f\left(\frac{b^{\prime}}{a^{\prime}}\right)\right| \geqslant \frac{c_{2}}{Q}
$$


for some constant $c_{2}>0$. Now the length of the interval $\left(p / q-c_{2} / Q, p / q+c_{2} / Q\right)$ is $2 c_{2} / Q$ and so by (13),

$$
\left|(\alpha, \beta) \cap \bigcup_{p / q}\left(\frac{p}{q}-\frac{c_{2}}{Q}, \frac{p}{q}+\frac{c_{2}}{Q}\right)\right| \geqslant \nu(\alpha, \beta) \frac{c_{2}}{Q} \geqslant c_{1} c_{2}(\beta-\alpha)
$$

by (12). Since $A=\bigcup_{r=1}^{\infty}\left(\alpha_{r}, \beta_{r}\right)$ and the $\left(\alpha_{r}, \beta_{r}\right)$ are disjoint,

$$
\begin{aligned}
\left|A \cap \bigcup_{p / q}\left(\frac{p}{q}-\frac{c_{2}}{Q}, \frac{p}{q}+\frac{c_{2}}{Q}\right)\right| & =\left|\bigcup_{r=1}^{\infty}\left(\alpha_{r}, \beta_{r}\right) \cap \bigcup_{p / q}\left(\frac{p}{q}-\frac{c_{2}}{Q}, \frac{p}{q}+\frac{c_{2}}{Q}\right)\right| \\
& =\sum_{r=1}^{\infty}\left|\left(\alpha_{r}, \beta_{r}\right) \cap \bigcup_{p / q}\left(\frac{p}{q}-\frac{c_{2}}{Q}, \frac{p}{q}+\frac{c_{2}}{Q}\right)\right| \\
& \geqslant \sum_{r=1}^{r_{0}}\left|\left(\alpha_{r}, \beta_{r}\right) \cap \bigcup_{p / q}\left(\frac{p}{q}-\frac{c_{2}}{Q}, \frac{p}{q}+\frac{c_{2}}{Q}\right)\right| \\
& \geqslant \sum_{r=1}^{r_{0}} c_{1} c_{2}\left(\beta_{r}-\alpha_{r}\right) \geqslant c_{1} c_{2} \frac{|A|}{2}=K|A|,
\end{aligned}
$$

where $K=c_{1} c_{2} / 2$ is independent of $Q, A$, as claimed.

Lemma 3. The set of rationals $p / q \in[\sqrt{15} / 8, \sqrt{3} / 2]$, where $p, \sqrt{q^{2}-p^{2}}, q$ is a Pythagorean triple, is ubiquitous with respect to the weight $\lfloor p / q\rfloor=q$ and the function $\rho: \mathbb{N} \rightarrow \mathbb{R}^{+}$given by $\rho(N)=c_{2} / N$, where $c_{2}$ is given by (13).

Proof. Let $A$ be an open subset of $[\sqrt{15} / 8, \sqrt{3} / 2]$. Take the resonant points $R_{j}$ to be $p / q$ with $\lfloor j\rfloor=q$. Then $\operatorname{dim} R_{j}=\operatorname{dim} \mathscr{R}=0, \operatorname{codim} \mathscr{R}=1$,

$$
B\left(R_{j} ; \rho(\lfloor j\rfloor)\right)=\left(\frac{p}{q}-\frac{c_{2}}{q}, \frac{p}{q}+\frac{c_{2}}{q}\right)
$$

and

$$
\left|A \cap \bigcup_{\lfloor j\rfloor=1}^{Q} B\left(R_{j} ; \rho(Q)\right)\right|=\left|A \cap \bigcup_{p / q}\left(\frac{p}{q}-\frac{c_{2}}{Q}, \frac{p}{q}+\frac{c_{2}}{Q}\right)\right| \geqslant K|A|,
$$

which satisfies the ubiquity condition (9), whence the family $\{p / q\}$ is ubiquitous with respect to $\rho: N \mapsto c_{2} / N$.

Let $\Psi(q)=q^{-v-1}$. Then $\Lambda(\mathscr{R} ; \Psi)$ is the set of points $u \in[\sqrt{15} / 8, \sqrt{3} / 2]$ such that

$$
\max \left\{\left|u-\frac{p}{q}\right|,\left|\sqrt{1-u^{2}}-\frac{r}{q}\right|\right\}<q^{-v-1}
$$

holds for infinitely many $p, q \in \mathbb{N}, r \in \mathbb{Z}$. It is readily verified that

$$
\Lambda(\mathscr{R} ; \Psi) \subset S_{v},
$$

whence $\operatorname{dim} S_{v} \geqslant \operatorname{dim} \Lambda(\mathscr{R} ; \Psi)$. But by Theorem 2 ,

$$
\operatorname{dim} \Lambda(\mathscr{R}, \Psi) \geqslant 0+\limsup _{N \rightarrow \infty} \frac{\log N^{-1}}{\log N^{-1-v}}=\frac{1}{v+1} .
$$


It follows from (7) and (8) that the dimension of $\mathscr{S}_{v}\left(\mathbb{S}^{1}\right)$ is $1 /(1+v)$ and Theorem 2 is proved.

In [14], the function corresponding to $\rho$ arises from Dirichlet's theorem on simultaneous Diophantine approximation and its value at $N$ is comparable to $N^{\frac{1}{2}}$. This gives rise to the factor $\frac{1}{2}$ in the lower bound in (4).

Note added in proof. Using lemma 4.1.1 in M. Huxley's book Area, lattice points and exponential sums (OUP 1996), it can be shown that $\operatorname{dim} \mathscr{S}_{v}\left(\mathbb{S}^{1}\right) \leqslant(2-v) /(1+v)$ for $1 / 2 \leqslant v \leqslant 1$. Sanju Velani has pointed out that this estimate can also be obtained more directly using $r(n)<4 d(n)<n^{\epsilon}$, where $r(n)$ is the number of integer solutions of the Diophantine equation $x^{2}+y^{2}=n$ and $d(n)$ is the divisor function.

Acknowledgements. We are grateful to Alan Baker, Victor Beresnevich, Basil Bernik, Jason Levesley and Andy Pollington for helpful discussions and suggestions, to the referee for pointing out a number of slips and obscurities and to EPSRC for financial support (grant reference GR/K56407).

\section{REFERENCES}

[1] A. Baker. A concise introduction to number theory (Cambridge University Press, 1984).

[2] A. Baker and W. M. Schmidt. Diophantine approximation and Hausdorff dimension. Proc. Lond. Math. Soc. 21 (1970), 1-11.

[3] R. C. Baker. Dirichlet's theorem on Diophantine approximation. Math. Proc. Camb. Phil. Soc. 83 (1978), 37-59.

[4] V. I. BerniK and M. M. Dodson. Metric Diophantine approximation on manifolds. Cambridge Tracts in Mathematics no. 137 (Cambridge University Press, 1999).

[5] H. Dickinson and M. M. Dodson. Extremal manifolds and Hausdorff dimension. Duke Math. J., 101 (2000), 271-281.

[6] M. M. Dodson, B. P. Rynne and J. A. G. Vickers. Metric Diophantine approximation and Hausdorff dimension on manifolds. Math. Proc. Cam. Phil. Soc. 105 (1989), 547-558.

[7] M. M. Dodson, B. P. Rynne and J. A. G. Vickers. Diophantine approximation and a lower bound for Hausdorff dimension. Mathematika, 37 (1990), 59-73.

[8] M. M. Dodson, B. P. Rynne and J. A. G. Vickers. Khintchine-type theorems on manifolds Acta Arith. 57 (1991), 115-130.

[9] M. M. Dodson, B. P. Rynne and J. A. G. Vickers. Simultaneous Diophantine approximation and asymptotic formulae on manifolds. J. Number Theory 58 (1996), 298-316.

[10] K. Falconer. The geometry of fractal sets. Cambridge Tracts in Mathematics 99 (Cambridge University Press, 1985).

[11] G. H. Hardy and E. M. Wright. An introduction to the theory of numbers (Clarendon Press, 4th edition, 1960).

[12] D. Y. Kleinbock and G. A. Margulis. Flows on homogeneous spaces and Diophantine approximation on manifolds. Ann. Math. 148 (1998), 339-360.

[13] P. MatTila. Geometry of sets and measures in Euclidean space (Cambridge University Press, 1995).

[14] Y. V. Mecnichuk. Diophantine approximation on curves and Hausdorff dimension. Dokl. Akad. Nauk Ukrain. SSR Series A, 9 (1978), 793-796.

[15] B. P. Rynne. Regular and ubiquitous systems, and $\mathscr{M}_{\infty}^{s}$-dense sequences. Mathematika 39 (1992), 234-243. 